University of Nebraska - Lincoln

DigitalCommons@University of Nebraska - Lincoln

Ralph Skomski Publications

Research Papers in Physics and Astronomy

April 1997

Domain-wall curvature and coercivity in pinning type $\mathrm{Sm}-\mathrm{Co}$ magnets

\author{
Ralph Skomski \\ University of Nebraska-Lincoln, rskomski2@unl.edu
}

Follow this and additional works at: https://digitalcommons.unl.edu/physicsskomski

Part of the Physics Commons

Skomski, Ralph, "Domain-wall curvature and coercivity in pinning type Sm-Co magnets" (1997). Ralph Skomski Publications. 27.

https://digitalcommons.unl.edu/physicsskomski/27

This Article is brought to you for free and open access by the Research Papers in Physics and Astronomy at DigitalCommons@University of Nebraska - Lincoln. It has been accepted for inclusion in Ralph Skomski Publications by an authorized administrator of DigitalCommons@University of Nebraska - Lincoln. 


\title{
Domain-wall curvature and coercivity in pinning type Sm-Co magnets
}

\author{
Ralph Skomski \\ Max-Planck-Institut für Mikrostrukturphysik Weinberg 2, 06120 Halle, Germany
}

The origin of coercivity in precipitation hardened $\mathrm{Sm}-\mathrm{Co}$ magnets is investigated. By considering domain-wall pinning at boundary phase and $\mathrm{Z}$ phase inhomogenities, it is found that the hexagonal $\mathrm{Z}$ phase has no direct influence on the coercivity. The intuitive assumption of plane domain walls made in a recent calculation is unrealistic but has no direct effect on the coercivity. However, due to the comparatively large size of the 12:17 cells, the calculated three dimensional domain-wall curvature is sufficiently large to assure a nearly ideal nestling of the domain walls to the 1:5 cell boundary. This nestling yields a coercivity increase from about 0.1 to about $1 \mathrm{~T}$ and is therefore responsible for the high coercivity of 2:17 based Sm-Co magnets. () 1997 American Institute of Physics. [S0021-8979(97)43708-8]

\section{INTRODUCTION}

The samarium-cobalt intermetallics $\mathrm{SmCo}_{5}$ and $\mathrm{Sm}_{2} \mathrm{Co}_{17}$ are appreciated for their high Curie temperatures, 1003 and $1190 \mathrm{~K}$, respectively, which are much higher than those of rare-earth iron permanent magnets. ${ }^{1-4} \mathrm{SmCo}_{5}$ is characterized by a very high magnetocrystalline anisotropy, $K_{1} \approx 17 \mathrm{MJ} / \mathrm{m}^{3}$, which makes it possible to produce nucleation controlled $\mathrm{SmCo}_{5}$ magnets. ${ }^{3}$ A disadvantage of $\mathrm{SmCo}_{5}$ is the comparatively low spontaneous magnetization, $\mu_{0} M_{s}=1.07 \mathrm{~T}$, since the energy product, a key figure of merit in permanent magnetism, increases quadratically with $M_{s}$. By comparison, the room temperature saturation magnetizations of $\mathrm{Nd}_{2} \mathrm{Fe}_{14} \mathrm{~B}$ and $\mathrm{Sm}_{2} \mathrm{Co}_{17}$ are 1.61 and $1.17 \mathrm{~T}$, respectively. Thus, from the point of view of saturation magnetization, $\mathrm{Sm}_{2} \mathrm{Co}_{17}$ is superior to $\mathrm{SmCo}_{5}$, but the comparatively low magnetocrystalline anisotropy of $\mathrm{Sm}_{2} \mathrm{Co}_{17}$, $K_{1}=3.3 \mathrm{MJ} / \mathrm{m}^{3}$, makes it difficult to create coercivity in pure $\mathrm{Sm}_{2} \mathrm{Co}_{17}$ magnets. $^{3}$

The outcome of the technological development of Sm-Co 2:17 magnets have been pinning, type hybrids where $\mathrm{Sm}_{2} \mathrm{Co}_{17}$ crystallites are surrounded by a $\mathrm{SmCo}_{5}$ boundary phase (Fig. 1). ${ }^{1,2}$ The $\mathrm{Sm}_{2} \mathrm{Co}_{17}$ cells, whose size is of order $100 \mathrm{~nm}$, are responsible for the saturation magnetization and consist of hexagonal $\mathrm{Th}_{2} \mathrm{Ni}_{17}$-type platelets in a rhombohedral $\mathrm{Th}_{2} \mathrm{Zn}_{17}$-type matrix. Since the wall energy $\gamma$ $=4 \sqrt{K_{1} A}$ is largest in the boundary phase, the $\mathrm{CaCu}_{5}$ type $1: 5$ regions enhance the coercivity by acting as pinning centers for the domain walls. ${ }^{2}$ In practice, the volume fractions of the 1:5 boundary and hexagonal 2:17 platelet phases are about $10 \%$ each. Here the fraction of the boundary phase is tuned by the samarium content, because the precipitation of the 1:5 phase requires some excess samarium. ${ }^{2}$

The nominal composition of optimum pinning type Sm-Co magnets, about $\mathrm{Sm}_{12} \mathrm{Co}_{58} \mathrm{Fe}_{24} \mathrm{Cu}_{4} \mathrm{Zr}_{2}$, ${ }^{5}$ indicates the importance of nonmagnetic additions such as $\mathrm{Cu}$ and $\mathrm{Zr}$. Experiments show that the addition of zirconium yields an increase of the coercivity of typical materials from less than 0.4 to more than $0.8 \mathrm{~T}^{5}$ Microstructurally, the zirconium atoms enter the hexagonal platelet or $\mathrm{Z}$ phase.

The question arises, whether the addition of $\mathrm{Z}$ phase merely modifies the phase-ordering kinetics ${ }^{2}$ or essentially contributes to the magnetic behavior of the material. ${ }^{6}$ On the one hand, it has been found that the hexagonal platelets are necessary for the formation of the 1:5 boundary phase, very likely by acting as easy diffusion pathways. ${ }^{2,4}$ On the other hand, it has been argued that there might be an additional pinning of plane domain walls where the zirconium rich hexagonal platelet phase intersects the boundary phase. ${ }^{6}$ In this article we will calculate the domain-wall curvature and show how the coercivity is affected by deviations from the planar wall shape.

\section{DOMAIN-WALL CURVATURE}

Micromagnetic and quantum-mechanical problems are similar in the sense that unknown magnetization and wave functions, respectively, have to be determined from a differential equation. ${ }^{7}$ If one knows the qualitative behavior of the function in question, then one can use suitable, parameter containing trial functions to describe the system. As it is well known from quantum mechanics, the optimum parameters are obtained by minimizing the energy whose functional derivative is the differential equation of the problem.

In the present context, the magnetization $\mathbf{M}(\mathbf{r})$ is given by the position of the domain wall, and we have to consider the (free) energy ${ }^{8}$

$$
E=-2 \mu_{0} M_{s} H \int_{\uparrow} d V+\int \gamma d S .
$$

Here $H$ is the magnetic field, applied along the crystallographic $c$ axis, $\gamma=4 \sqrt{K_{1} A}$ is the domain-wall energy, and the index $\uparrow$ indicates that the volume integration includes all regions where magnetization and external field are parallel.

Let us first consider a plane wall parallel to the crystallographic $c$ axis, whose position can be parametrized by a

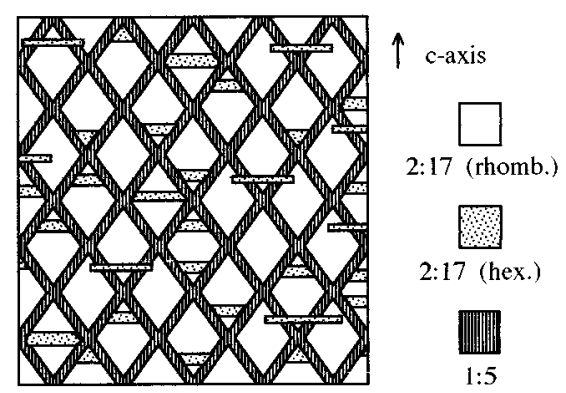

FIG. 1. Schematic microstructure of a pinning type $\mathrm{Sm}_{2} \mathrm{Co}_{17}$ magnet. 


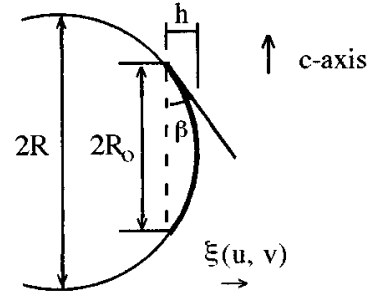

FIG. 2. A domain wall having the curvature of a sphere of radius $R$. The dashed line shows the position of the $\xi_{0}=0$ plane.

displacement parameter $\xi$. Equation (1) then becomes $E=-2 \mu_{0} M_{s} H L^{2} \xi+\langle\gamma(\xi)\rangle L^{2}$, where $L^{2}$ is the wall area, and $\langle\ldots\rangle$ denotes the average over the wall area. For $H=0$, the energy minimum is given by the position $\xi_{0}=0$ at which the average wall energy $\langle\gamma\rangle$ is lowest. If the domain wall is curved, then the displacement depends on the position of the wall element: $\xi=\xi(u, v)$. Here $u$ and $v$ are Cartesian coordinates so that $\xi(0,0)$ indicates the center of the cell. In terms of $\xi(u, v)$, the energy is

$$
\begin{aligned}
E= & -2 \mu_{0} M_{s} H \int \xi(u, v) d u d v \\
& +\gamma \int \sqrt{1+\left(\frac{\partial \xi}{\partial u}\right)^{2}+\left(\frac{\partial \xi}{\partial v}\right)^{2}} d u d v .
\end{aligned}
$$

Since the wall is pinned at the boundaries of the cell, the function $\xi\left(u_{i}, v_{i}\right)$ equals zero at each point $i$ of the cell boundary.

To calculate the curvature of the wall, we approximate the wall inside a given cell by a segment of a sphere of radius $R$ (Fig. 2). This implies $R^{2}=\sqrt{(\xi-\lambda)^{2}+u^{2}+v^{2}}$, where the parameter $\lambda$ is necessary to match the boundary condition $\xi=0$ at $u^{2}+v^{2}=R_{0}^{2}$. Here the quantity $2 R_{0}$ can be interpreted as some average cell size. Putting $\xi\left(u, v ; R, R_{0}\right)$ into Eq. (2) and minimizing $E$ with respect to $R$, we obtain after short calculation the surprisingly simple result $R=\gamma / \mu_{0} M_{s} H$. The angle $\beta$, defined in Fig. 2 , is given by

$$
\beta=\arcsin \frac{\mu_{0} M_{s} H R_{0}}{\gamma} .
$$

Taking $\mu_{0} M_{s}=1.17 \mathrm{~T}, \quad \gamma=35 \mathrm{~mJ} / \mathrm{m}^{2},{ }^{6}, R_{0}=40 \mathrm{~nm}$, and $\mu_{0} H=0.8 \mathrm{~T}$ yields $\beta=56^{\circ}$. This means that the domain-wall curvature inside the $2: 17$ cells is nonnegligible, in spite of the comparatively high domain-wall energy of $\mathrm{Sm}_{2} \mathrm{Co}_{17}$. This result agrees with experimental findings: Lorentz electron micrographs ${ }^{2}$ show that the domain walls adapt to the cell boundary characterized by an angle of about $30^{\circ}$.

\section{COERCIVITY}

\section{A. Influence of domain-wall curvature}

To investigate how the domain-wall curvature affects the coercivity, we have to add a pinning term $\Delta E=2 \pi R_{0} f\left(\xi_{0}\right)$ to the energy Eq. (2). The function $f\left(\xi_{0}\right)$ describes the dependence of the wall energy on the wall position at the cell boundary. Introducing the maximum displacement $h=R$ $-\sqrt{R^{2}-R_{0}^{2}}$ of the wall compared to the reference plane (Fig. $2)$, we can write the total energy as

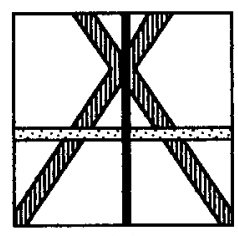

(a)

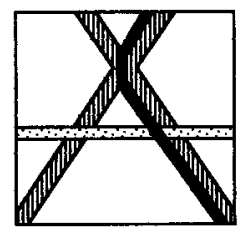

(c)

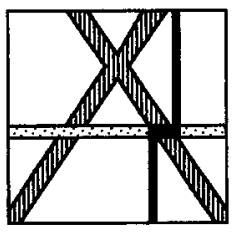

(b)

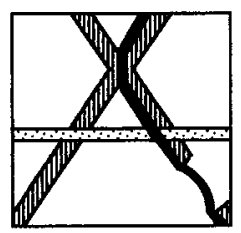

(d)
FIG. 3. Schematic wall configurations: (a) plane wall, (b) wall intersecting a $\mathrm{Z}$ phase platelet, (c) curved wall, and (d) imperfect wall.

$$
\begin{aligned}
\frac{E}{\pi}= & -2 \mu_{0} M_{s} H\left(\frac{h^{3}}{6}+\frac{h R_{0}{ }^{2}}{2}+R_{0}{ }^{2} \xi_{0}\right)+\sigma\left(R_{0}{ }^{2}+h^{2}\right) \\
& +2 R_{0} f\left(\xi_{0}\right) .
\end{aligned}
$$

Fortunately, the two trial parameters $h$ and $\xi_{0}$ are decoupled in this equation, so that minimizing $E$ with respect to $\xi_{0}$ yields after short calculation the pinning coercivity $H_{c}=\max \left(\partial f / \partial \xi_{0}\right) / \mu_{0} M_{s} R_{0}^{2}$. This coercivity is independent of the curvature radius $R$ so long as the wall does not touch the cell boundary.

\section{B. The variational nature of pinning coercivity}

In Ref. 6, the coercivity mechanism has been deduced by comparing the energies of several domain-wall configurations (Fig. 3). The main competition was assumed to be between ideal plane walls [Fig. 3(a)] and plane walls utilizing the comparatively low domain-wall energy in the hexagonal platelets [Fig. 3(b)]. The existence of pinning configurations of the type Fig. 3(b) has given rise to the conclusion that the $\mathrm{Z}$ phase gives a direct contribution to the coercivity. ${ }^{6}$ It is, however, not appropriate to compare total energies, since most domain-wall configurations of interest in permanent magnetism are metastable. In fact, both Figs. 3(a) and 3(b) represent local energy minima able to pin domain walls. Furthermore, the consideration of a single cell in Fig. 3(b) is not sufficient, since the position of the $\mathrm{Z}$ phase platelets is more or less random. In other words, the number of cells having the $Z$ phase platelets at the right position is limited, and the domain wall is likely to cross pure, energetically unfavorable 1:5 regions above and below the cutting shown in Fig. 3(b).

To estimate the plain-wall coercivity caused by the configuration of Fig. 3(a), we have to determine the pinning energy $f\left(\xi_{0}\right)$ or, alternatively, $\left\langle\gamma\left(\xi_{0}\right)\right\rangle=\langle\gamma(\xi)\rangle$. Since only differences in the domain-wall energies are of importance, the wall-energy difference between the 1:5 and 2:17 regions, $\Delta \gamma \approx 23 \mathrm{~mJ} / \mathrm{m}^{3}{ }^{6}$, is the key parameter. Now we make the fair assumption that the area of the intersected 1:5 phase doubles 
on moving the wall by $\xi_{0}=b$, where $b$ is the thickness of the separating boundary phase. This yields, after short calculation,

$$
H_{c}=\frac{\Delta \gamma \Phi}{2 \mu_{0} M_{s} b},
$$

where $\Phi$ is the volume fraction of the boundary phase. Taking $b=10 \mathrm{~nm}$ and $\Phi=10 \%$, we obtain $\mu_{0} H_{c} \approx 0.1 \mathrm{~T}$, which is one order of magnitude smaller than the experimental coercivity. Up to a numerical prefactor of order one, the same result is obtained for the configuration of Fig. 3(b).

In practice, the pronounced domain-wall curvature leads to configurations, such as that shown in Fig. 3(c). Here, the area contributing to the pinning is not restricted to a small fraction $\Phi$ of the wall, so that we can put $\Phi=1$ in Eq. (5). This yields coercivities of order $1 \mathrm{~T}$, in agreement with experiment.

It is important to note that this estimate remains true if the 1:5 boundary phase is defective. Consider, for example the case where the 1:5 boundary region contains a hole of radius $R_{0}=R_{h}$ [Fig. 3(d)]. Taking $\mu_{0} M_{s}=1.17 \mathrm{~T}, \gamma=35$ $\mathrm{mJ} / \mathrm{m}^{7},{ }^{6} \mu_{0} H=0.8 \mathrm{~T}$, and $R_{0}=10 \mathrm{~nm}$, we obtain from Eq. (3) the angle $\beta=12^{\circ}$. This means that the wall remains largely uncurved in the hole, the wall cannot expand like a bubble gum into the neighboring cell, and the coercivity is only slightly reduced by the defect.

\section{DISCUSSION}

From the point of view of mathematical physics, where magnetization states and coercivities are analoges to quantum-mechanical wave functions and energy values, respectively, the assumption of a domain-wall curvature amounts to the choice of a particular trial wave function. To model three dimensional domain walls, we have assumed that the curvature of the wall is constant everywhere, so that the wall represents a section of a sphere. This is, of course, an approximation, since the true shape of the wall depends on detail of the cell boundaries and cannot be calculated in a closed form. However, variational ground-state energies are generally more exact than ground-state eigenfunctions, so that the details of the wall shape are of minor importance in the present context.

Note the general rule that the size of the inhomogeneities, that is the thickness of the 1:5 regions, has to be comparable to the domain-wall thickness $\delta_{W} \approx 5 \mathrm{~nm}^{8,9}$ Much thinner boundary regions lead to a "tunneling" of the wall through the boundary phase.

\section{CONCLUSIONS}

In conclusion, we have calculated the domain-wall curvature in the 2:17 cells of pinning type Sm-Co magnets, which turns out to be very large. Although the coercivity is not affected by this curvature in lowest order, there is an indirect coercivity enhancement due to the fact that the curved domain walls touch the 1:5 boundary regions. The hexagonal $\mathrm{Z}$ phase platelets contribute to the formation of the cell boundaries and may act as pinning centers but are unlikely to yield a dominating contribution to the coercivity. In fact, even in the absence of $\mathrm{Z}$ phase platelets, the presence of a well developed 1:5 boundary phase would assure a high coercivity.

${ }^{1}$ J. D. Livingston and D. L. Martin, J. Appl. Phys. 48, 1350 (1977).

${ }^{2}$ F. Fidler, J. Magn. Magn. Mater. 30, 58 (1982); Wiss. Z. HfV Dresden, S 50, 24 (1989).

${ }^{3}$ Concise Encyclopedia of Magnetic and Superconducting Materials, edited by J. E. Evetts (Pergamon, Oxford, 1992).

${ }^{4}$ L. Rabenberg, R. K. Mishra, and G. Thomas, J. Appl. Phys. 53, 2389 (1982).

${ }^{5}$ R. A. McCurrie, Ferromagnetic Materials (Academic, London, 1994).

${ }^{6}$ M. Katter, J. Weber, W. Assmus, P. Schrey, and W. Rodewald, IEEE Trans. Magn. 32, 4815 (1996).

${ }^{7}$ R. Skomski and J. M. D. Coey, Phys. Rev. B 48, 15812 (1993).

${ }^{8}$ E. Kneller, Ferromagnetismus (Springer, Berlin, 1962).

${ }^{9}$ D. Sander, R. Skomski, C. Schmidthals, A. Enders, and J. Kirschner, Phys. Rev. Lett. 77, 2566 (1996). 